\title{
Cardiac Remodeling During the Morphogenesis of Neuroleptic Cardiomyopathy
}

\author{
Volkov VP* \\ Tver center of judicial examinations, Russia

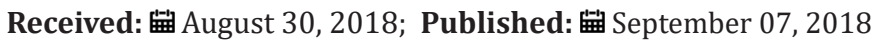 \\ *Corresponding author: Volkov VP, Tver center of judicial examinations, Russia
}

\begin{abstract}
Introduction: A neuroleptic cardiomyopathy NCMP belongs to secondary specific metabolic dilated cardiomyopathies. It is caused by side cardiotoxic effect of antipsychotic preparations. In its development NCMP passes three clinical stages: 1) latent, 2) developed and 3) terminal.
\end{abstract}

Aim: A morphometric study of the macroscopic heart condition in each clinical stage of NCMP.

Material and methods: The autopsy protocols of 80 dead persons with NCMP in various stages and of 100 persons who did not have any accompanying cardiac pathology were studied. For analysis of the received data the original author's method that we had developed for such studies was used.

Results: All macroscopic cardiac parameters in the case of NCMP statistically significantly differ from normal one, but no significant differences in various clinical stages of NCMP.

Conclusion: The process of cardiac remodeling on the organ level ends during the latent stage of the disease. Progression of myocardial dysfunction is connected with changes of myocardium microstructure.

Keywords: Neuroleptic cardiomyopathy; morphogenesis; remodeling of a heart; morphometric research

\section{Introduction}

Neuroleptic cardiomyopathy (NCMP) is one of serious vitally dangerous complications of psychotropic therapy, with is caused by a side cardiotoxic effect of antipsychotic drugs, as classical, and atypical [1-9]. This disease belongs to secondary specific metabolic dilated cardiomyopathies $[10,11]$ and is characterized by a diffuse myocardial involvement, by a drastic reduction of its contractile function and, as consequence, by a progressive congestive chronic heart failure (CHF) $[1,10,12,13]$. In its development NCMP passes three clinical stages: 1) a latent one, clinically fully compensated one, 2) a full-scale (developed. manifesting) one, when cardiac disorders are clearly detected, but without evident signs of CHF, and 3) a terminal one, when the clinical picture of CHF comes to the foreground $[10,14]$. As you know all changes of function of some or other organs, especially the heart, are initially caused by it structural changes which are a physical substrate of pathophysiological shifts and reflect the morphogenesis of a pathology [15]. During the morphogenesis of NCMP, the heart undergoes a remodeling process at the macroscopic (organ) level of the organization. As L a Bokeria and co-authors (2010) [16] point out, initially the remodeling of the heart in any pathology has a compensatory-adaptive nature, aimed at preserving the pumping function of the heart. However, with the development of the disease compensatory capabilities of the heart are exhausted, there is a failure of adaptation, and manifests myocardial dysfunction. But until now, a deep comparative morphological study of the macroscopic heart condition in each clinical stage of NCMP, including those associated with the process of remodeling, has not been conducted. Therefore, the aim of this study is to eliminate - at least in part - the existing gap.

\section{Material and Methods}

The autopsy protocols of 80 dead persons in the age from 16 to 77 years ( 60 men and 20 women) were studied, in which the NCMP 
was revealed by section. In 36 of these patients the disease was in the latent stage (group II), in 15 patients it was in the developed one (group III), and in 29 patients it was in the terminal one (group IV). As a control, we studied the autopsy protocols of 100 persons ( 50 men and 50 women) in the age from 18 to 82 years who died from non cardiac causes and who did not have any accompanying cardiac pathology and this fact was verified by autopsy (group I). The cardiac parameters, which were received in this group, are taken as a relative norm (RN).

The following parameters were measured on the macroscopic level: heart mass (m), linear dimensions, perimeter of venous valve openings, and thickness of a wall of ventricles. For analysis of the received data we used an original method that we had developed for such studies [17]. For this analysis the outer volume of heart without atria (V) was determined and two relative parameters (both in percent) were calculated: 1 ) $\mathrm{C}_{\mathrm{v}}$ - coefficient of volume, this coefficient shows a part of the total volume of heart (without atria), and this part falls on the volume of cavities of ventricles; and 2) $\mathrm{C}_{1}$ - coefficient of the left ventricle, this coefficient shows the volume size of the left ventricle with respect to the total volume of both ventricles. In addition, two other parameters were calculated which use a gravimetric characteristic of the heart $(\mathrm{m})$ : mass-volume ratio (MVR) and index of density of myocardium (IDM). A growth of MVR is evidence of a hypertrophy of myocardium, and its diminution is an indication for dilatation of cavities of heart ventricles. IDM clearly shows a strongly expressed correlation with such objective parameters of microstructure of cardiac muscle as stromalparenchymatous ratio and rate of interstitial edema [17], which quantitatively describe a condition of its intercellular matrix. The received results were statistically processed by the nonparametric Mann- Whitney's U-criterion with significance level of distinctions $95 \%$ and more $(\mathrm{p} \leq 0.05)$.

\section{Results}

Table 1: Macroscopic characteristic of heart during NCMP morphogenesis.

\begin{tabular}{|c|c|c|c|c|c|c|}
\hline Gr. & $\mathbf{m}$ & $\mathbf{V}$ & Kv & Kl & MVR & IDM \\
\hline I & 300 & 131.6 & 32.1 & 39.1 & 2.28 & 4.42 \\
\hline II & $\begin{array}{c}355 \\
1\end{array}$ & $\begin{array}{c}163.5 \\
1\end{array}$ & $\begin{array}{c}41.4 \\
1\end{array}$ & 40.2 & 2.17 & $\begin{array}{c}6.06 \\
1\end{array}$ \\
\hline III & $\begin{array}{c}358 \\
1\end{array}$ & $\begin{array}{c}165.8 \\
1\end{array}$ & $\begin{array}{c}42.6 \\
1\end{array}$ & 40.3 & $\begin{array}{c}2.16 \\
1\end{array}$ & $\begin{array}{c}6.24 \\
12\end{array}$ \\
\hline IV & $\begin{array}{c}361 \\
1\end{array}$ & $\begin{array}{c}167.5 \\
1\end{array}$ & $\begin{array}{c}43.2 \\
1\end{array}$ & $\begin{array}{c}40.6 \\
1\end{array}$ & $\begin{array}{c}2.15 \\
1\end{array}$ & $\begin{array}{r}6.36 \\
123\end{array}$ \\
\hline
\end{tabular}

Note: 1 - statistically significant distinctions with gr. I.

2 - statistically significant distinctions with gr. II.

3 - statistically significant distinctions with gr. III.
The results of research which has been conducted on the organ level are presented in the Table 1. As follows from its analysis, all macroscopic cardiac parameters in the case of NCMP statistically significantly differ from RN. At the same time, no significant differences of $\mathrm{m}, \mathrm{V}$ and MVR were found in various clinical stages of NCMP. The dynamics of value $C_{v}$ have the same character. At the same time, the values $\mathrm{C}_{1}$ differs from $\mathrm{RN}$ only in the terminal stage of NCMP, and to a significantly lesser degree than $\mathrm{C}_{\mathrm{v}}$. Changes of IDM are the most important ones. The values of this parameter grow already in the latent stage of NCMP (group II) and steadily progress later on.

\section{Discussion}

On the basis of analysis of detected cardiac changes, which take place in the process of remodeling of the heart in different clinical stages of NCMP, the morphogenesis of the disease seems to be the following. In the case of NCMP the cardiomegaly develops already during the latent stage of the disease and does not distinctly progress subsequently. This is proved by the absence of significant differences of $\mathrm{m}, \mathrm{V}$ and MVR in different clinical stages of NCMP. At the same time, changes of $\mathrm{C}_{\mathrm{v}}$ and $\mathrm{C}_{1}$ tell about a uniform dilatation of both heart ventricles with some predominance of the left one, only in the late phases of NCMP morphogenesis (terminal stage). On the contrary, an initially progressive growth of IDM tells about the developing damages of myocardium microstructure, in particular, about changes of its intercellular matrix.

Thus, the received data are evidence of the fact that the process of the cardiac remodeling, which takes place in the course of NCMP development on the organ level, ends when the disease passes to the full-scale stage. Further progression of a myocardial dysfunction leads to the development of $\mathrm{CHF}$ and is determined by the growing changes of microstructure of cardiac muscle. In other words, appearance of clinical manifestations of NCMP is accompanied by transition of cardiac remodeling to the deeper (tissue and cellular) organizational levels. This thesis is confirmed and convincingly proved by results of morphometric research of myocardium microstructure changes in various stages of NCMP morphogenesis, which correspond to the clinical stages of the disease course [1820]. Though, as they say, it is a completely different story.

\section{Conclusion}

The conducted research has proved that in the course of NCMP morphogenesis cardiac remodeling on the organ level is observed. In the latent stage of the disease the macroscopic cardiac remodeling reflects the compensatory-adaptive processes which are directed towards preservation of the pumping function of a heart. The full-scale stage of NCMP is characterized by termination of remodeling on the organ level, and the further progression of myocardial dysfunction, which is to the maximum expressed in the 
terminal stage of the disease, takes place because of the pathologic changes of myocardium.

\section{References}

1. Volkov V (2018) Cardiotoxicity of neuroleptics: clinical aspects. Beau Bassin: LAP Lambert Academic Publ.

2. Volkov VP (2010) Cardiotoxicity of phenothiazine neuroleptics (review of literature). Psichiat psychopharmacother 12(2): 41-45.

3. Buckley NA, Sanders P (2000) Cardiovascular adverse effects of antipsychotic drugs. Drug Saf 23(3): 215-228.

4. Coulter DM, Bate A, Meyboom RHB, Lindquist M, Edwards IR (2001) Antipsychotic drugs and heart muscle disorder in international pharmacovigilance: data mining study. Br Med J 322(7296): 1207-1209.

5. Curto M, Girardi N, Lionetto L, Ciavarella GM, Ferracuti S, et al. (2016) Systematic review of clozapine cardiotoxicity. Curr Psychiatry Rep 18(7): 68.

6. Khasawneh FT, Shankar GS (2014) Minimizing cardiovascular adverse effects of atypical antipsychotic drugs in patients with schizophrenia. Cardiol Res Pract pp. 273060.

7. Mackin P (2008) Cardiac side effects of psychiatric drugs. Hum Psychopharmacol 23(Suppl 1): 3-14.

8. Potočnjak I, Degoricija V, Vukičević Baudoin D, Čulig J, Jakovljević M (2016) Cardiovascular side effects of psychopharmacologic therapy. Int J Cardiol 219: 367-372.

9. Raedler TJ (2010) Cardiovascular aspects of antipsychotics. Curr Opin Psychiatry 23(6): 574-581.
10. Volkov VP (2013) Clinical characteristic of an antipsychotic cardiomyopathy. In VP Volkov (ed.) Actual problems of therapeutic clinic: collective scientific monograph. Novosibirsk: SibAC pp. 94-116.

11. Tereshchenko SN, Jaiani NA (2001) Dilated cardiomyopathy today. Zhurn serdechnaya nedostatochnost 3(2): 58-60.

12. Volkov VP (2009) Phenothiazine dilated cardiomyopathy: some aspects of clinic and morphology. Klin Med 87 (8): 13-16.

13. Volkov VP (2011) To a question of a secondary phenothiazine cardiomyopathy. Klin Med 89(5): 30-33.

14. Volkov VP (2011) Features of the electrocardiogram at a phenothiazine cardiomyopathy. Klin Med 89(4): 27-30.

15. Sarkisov DS, Paltsev MA, Hitrov NK (1995) General pathology of the person. Moscow: Medicine Publ.

16. Bokeriya LA, Bokeriya OL, Le TG (2010) Electrophysiological myocardial remodeling in heart failure and various heart diseases. Ann aritmol $7(4): 41-48$.

17. Volkov VP (2014) New method of an organometry of heart. In: VP Volkov (ed.) Paraclinical disciplines: new methods and diagnostic opportunities: the collective monograph. Novosibirsk: SiBAK Publ pp. 78-100.

18. Volkov VP (2018) Cardiotoxicity of neuroleptics: essaies of the study of the problem. Beau Bassin LAP Lambert Academic Publ.

19. Volkov VP (2018) Morphometric approach to the study of morphogenesis of a neuroleptic cardiomyopathy. Glob J Add \& Rehab Med 5(2): 555658.

20. Volkov VP (2012) Morphometric aspects of a morphogenesis of an antipsychotic cardiomyopathy. Ros J Cardiol 3: 68-73.
To Submit Your Article Click Here:

This work is licensed under Creative Commons Attribution 4.0 License

Submit Article

DOI: 10.32474/ACR.2018.01.000115

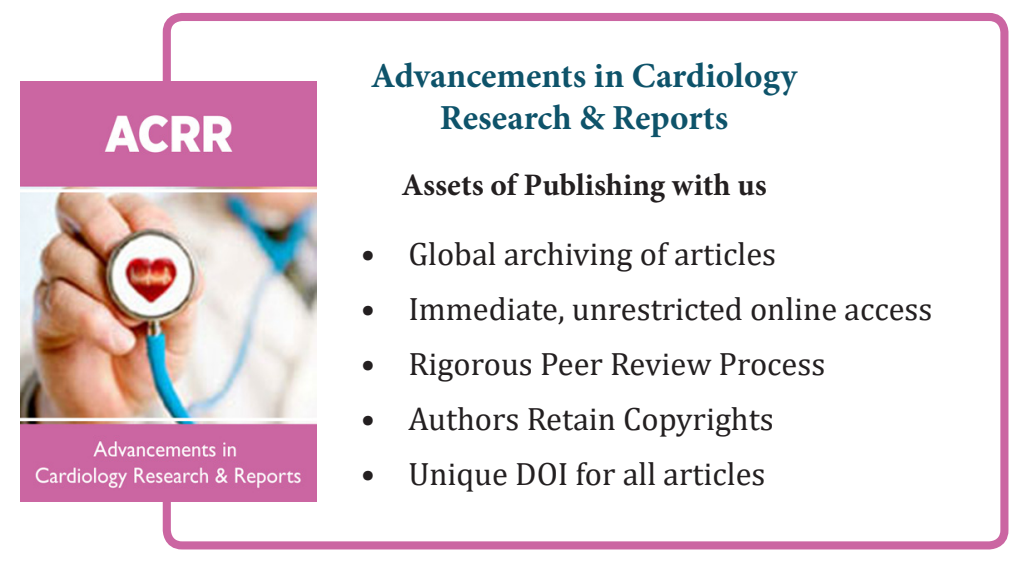

